\title{
Contact Fatigue Failure of Ultra- High Molecular Weight Polyethylene Bearing Components of Knee Prostheses
}

The objective of this work has been to study the origin of surface failures in UHMWPE tibial bearings of total knee replacements. Earlier examination of hundreds of retrieved prostheses, along with analysis of the properties of UHMWPE material from retrieved bearings, had shown that a large number of the bearings suffered from subsurface oxidation. This oxidation was related to the gamma irradiation used to sterilize the tibial bearings. Mechanical properties of the polymer were significantly deteriorated in the oxidized region, with the most severe reduction of strength and ductility occurring about $1 \mathrm{~mm}$ beneath the contact surface. In this work the contact stress distribution in the bearings was analyzed, and tribotesting of the bearing materials was carried out under simulated service conditions. Fatigue cracks and delamination developed in bearings tested in a knee simulator and in rolling/sliding test specimens, and the damage was similar to that found in retrieved tibial bearings. The fatigue cracks invariably initiated in the embrittled oxidized layer, and the depth of that layer determined the depth at which the cracks began. The stress analysis showed that the maximum shear stress and von Mises equivalent stress reached high levels in the subsurface oxidized zone where the delamination and contact fatigue failures initiated.
J. L. Duda

D. P. Gestwick

J. P. Collier

B. H. Currier

Thayer School of Engineering, Dartmouth College, Hanover, NH 03755

M-C. Dubourg

Labo. de Mécanique des Contacts, INSA de Lyon,

69621 Villeurbanne, France

\section{Introduction}

Arthritis of the knee is a crippling disease which can severely limit the mobility of those afflicted by it. Joint replacement represents an appealing means to relieve the pain and increase the mobility of arthritic patients. Nearly 200,000 Total Knee Replacements (TKR) are implanted annually in the United States (Cameron, 1994). Most TKR designs incorporate a polished metallic femoral component, usually made from a cobaltchrome alloy, in contact with a tibial bearing made from UltraHigh Molecular Weight Polyethylene (UHMWPE). An example of a typical TKR design is shown in Fig. 1. In contrast to a total hip replacement, which consists of a conformal ball-in-socket joint, the bearing in a TKR is primarily nonconformal and features a relatively concentrated contact in most designs. Whereas the kinematic contact conditions in a hip joint result in multidirectional oscillatory sliding, those in the knee are a combination of oscillatory rolling and sliding. The loading conditions in knee joints are cyclic in nature, and they vary with the flexion and extension angle. One model of the loads seen by a knee during a typical walk cycle is shown in Fig. 2 (ISO, 1996). Owing to inertia forces during the human gait, loads of more than 3-4 times body weight can be supported by the knee during a typical walk cycle (Fig. 2), and other research has shown that loads can exceed 6 times body weight in many cases (Seireg and Arvikar, 1975). These loads cause high contact pressures in the concentrated contact between the metallic femoral component and the UHMWPE tibial bearing (McNamara et al., 1994). Therefore, after implantation the nonconforming TKR bearings are subjected to many cycles of high contact stress during oscillatory rolling/sliding contact.

Although many implanted knee prostheses have withstood $>10$ or even 20 years of activity without substantial surface damage (Fig. 3(a)), many other tibial bearings of TKR have shown evidence of significant wear and contact fatigue damage, requiring early replacement $(<5$ years after implantation) (Fig. 3(b)). The replacement procedures are costly and can have serious health consequences.

The objectives of this work have been to study the factors contributing to surface failures in UHMWPE tibial bearing components of total knee replacements.

\section{Background}

Ultra-high molecular weight polyethylene (UHMWPE) has been used as the primary bearing material in orthopaedic implants for more than 30 years. In most total knee replacements the tibial bearings are made from medical grade UHMWPE having an average molecular weight of 4 million or greater; they are either molded or machined to their final shape. Before the TKR components can be implanted in humans they must be sterilized, and until recently nearly all UHMWPE bearings used in the United States had been sterilized with gamma irradiation in air.

However, studies of bearings explanted from patients have 


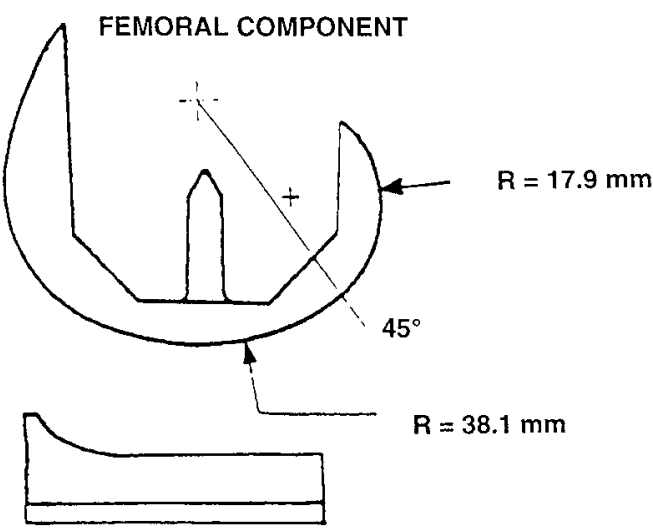

TIBIAL COMPONENT

Fig. $1(a)$

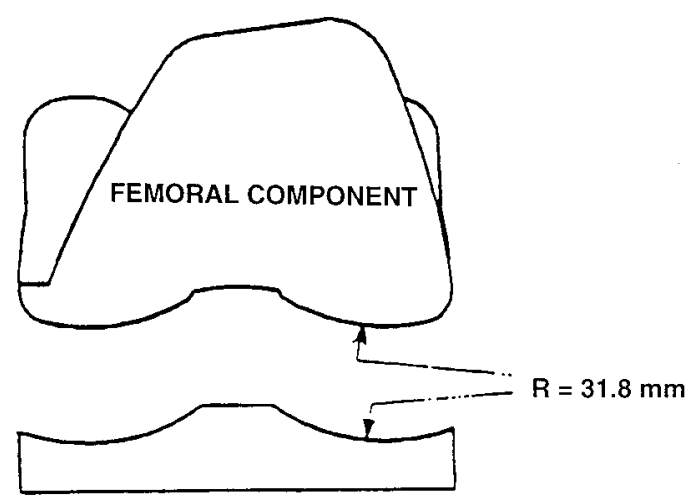

TIBIAL COMPONENT

Fig. $1(b)$

Fig. 1 Diagram of femoral and tibial components of a typical condylartype total knee prosthesis. (a) Side view. (b) Front view.

shown dramatic variations in polyethylene wear and fatigue damage. Historically, these variations were attributed in part to patient variables, component designs, and in-vivo service histories (Bartel et al., 1986; Landy and Walker, 1988). Recent research has demonstrated that the damage seen in many explanted polyethylene bearings can be linked to sterilization by gamma irradiation in air and subsequent material degradation (Sutula et al., 1995; Collier et al., 1996).

Over 1600 retrieved UHMWPE knee bearings, including both patellar and tibial bearings, have been examined in recent years at Dartmouth Biomedical Engineering Center (Williams et al., 1998). Those components were provided by about 300 surgeons from
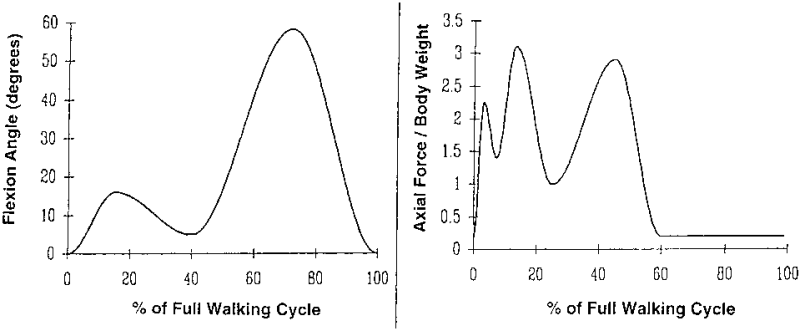

Fig. 2 Load and flexion angle variations in knee during a typical walk cycle (from ISO, 1996)

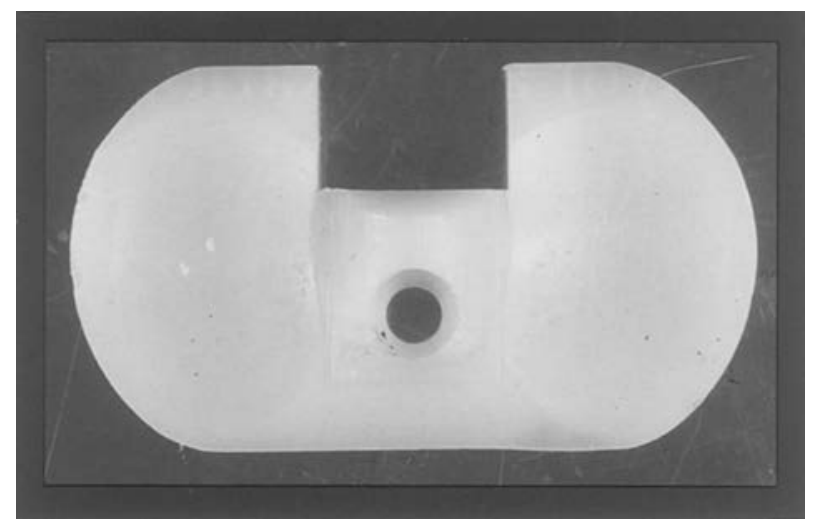

Fig. 3(a) Retrieved UHMWPE tibial bearing which successfully served the patient with little visible surface damage

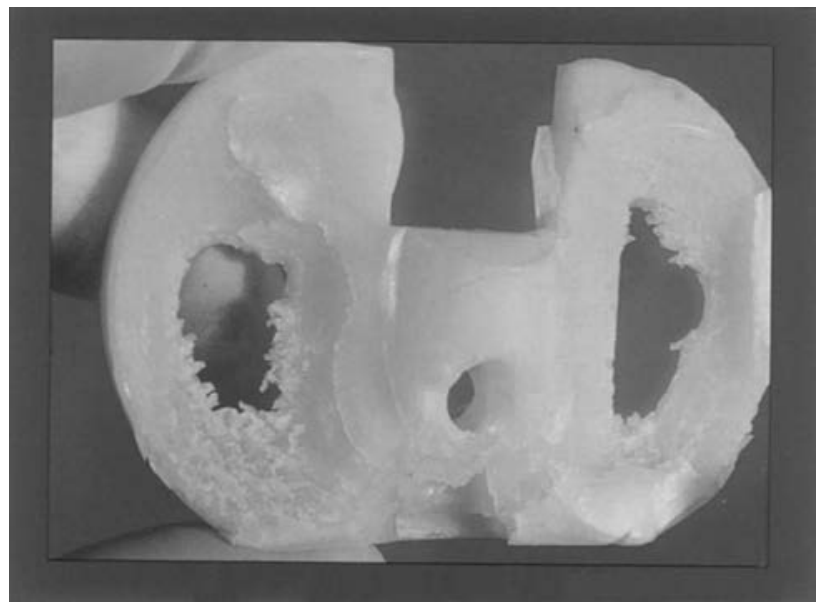

Fig. 3(b) Retrieved tibial bearing which experienced catastrophic surface damage in the form of cracking and delamination within 8 years of service

North America and Europe, and they had been produced by a dozen different manufacturers. The bearings had been removed either during revision surgery or post-mortem and had been in service in-vivo for an average of about 5 years, with a range of 1 month to 20 years. Examination of the knee bearings showed that a variety of wear modes, including abrasion, burnishing and scratching, were observed on bearing surfaces, including those bearings with short in-vivo duration. The most prevalent surface failure mechanisms, however, particularly among those components that had been in service for a longer time, were delamination and contact fatigue cracking. 55\% of the retrieved bearings had indications of delamination or cracking or both (Williams et al., 1998), and in nearly all cases the damage appeared to initiate subsurface. The incidence of damage was highest in bearings that had been sterilized with gamma radiation; 902 of the 1603 gammairradiated bearings showed cracking or delamination or both. The small number $(n=32)$ of knee bearings that were known to have been sterilized by an alternative technique (ethylene oxide, or EtO) showed no evidence of contact fatigue damage, even after in-vivo durations in excess of 15 years (Williams et al., 1998). When the specimen population was limited to gamma-irradiated components that had been in-vivo for more than four years, the percentage of tibial bearings showing either delamination or cracking increased to $>65 \%$ (Collier et al., 1996) or up to $80 \%$ (Williams et al., 1998). The damage was so severe that about $20 \%$ of the bearings were worn through their entire thickness (Fig. 3(b)). These results are similar to those of Blunn et al. (1997), who reported wear comparisons for 280 retrieved bearings of eight different designs. Delamination was seen in retrievals of all designs except one and 


\section{OXIDATION PROFILE}

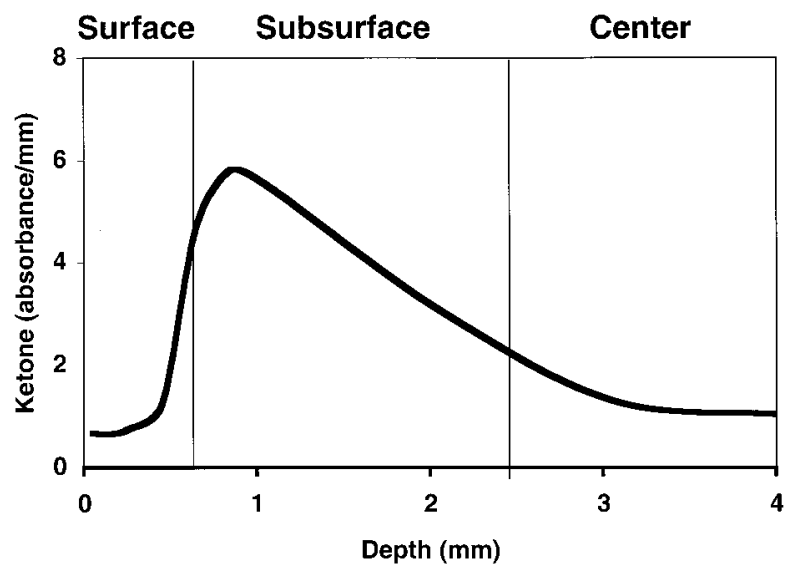

Fig. 4 FTIR-measured oxidation level (ketone peak absorbance level/ thickness in $\mathrm{mm}$ ) versus depth (microns) for a never-implanted polyethylene tibial component $\mathbf{8}$ years after gamma sterilization in air

in the four knee designs for which delamination frequency was reported, 65 per cent of bearings showed delamination.

About 400 of the retrieved UHMWPE bearings in the Dartmouth study were sliced through their thickness and then thin sections (200 $\mu \mathrm{m}$ thick) were cut from the cross-sections using a microtome. Microtomed thin sections of retrieved tibial bearing components showed that $68 \%$ of the sections from bearings that had been gamma irradiated in air had a subsurface white band (Williams et al., 1998). The white band was present in approximately $90 \%$ of the bearings that had cracked or delaminated in service (Collier et al., 1996), so there was a very strong association between the presence of a subsurface white band and contact fatigue damage. Subsequent analysis of the white band using Fourier Transform Infrared Spectroscopy (FTIR) showed that it was characterized by oxidized polyethylene (Collier et al., 1996). The location of peak oxidation was $1-1.5 \mathrm{~mm}$ subsurface and was within the white band. Figure 4 shows a typical oxidation profile for specimens which had been gamma irradiated in air and shelf aged. Oxidation is measured by the maximum level of absorbance at the ketone peak $\left(1717 \mathrm{~cm}^{-1}\right)$, and that peak absorbance value is then divided by the specimen thickness ( $\mathrm{mm})$. The material thickness is roughly separated into three regions, a surface 'clear' layer, a subsurface oxidized ('white') layer, and a central region. The oxidation profile is symmetrical about the centerline of the material, and only the top half of the thickness is shown in Fig. 4. (Note: The layer boundaries shown on Fig. 4 are only approximate; there is no sharp demarcation between the layers.) The surface layer was clear and showed much less oxidation than the subsurface white layer; the center of the bearing thickness also showed relatively low oxidation. The oxidation takes time to occur, so the oxidation level is a function of the environment in which the bearing was irradiated and stored, and the length of time after irradiation (Sutula et al., 1995). Specimens which had been sterilized using EtO had significantly lower oxidation levels (Williams et al., 1998).

Mechanical testing was done on dumbbell-shaped test specimens [ASTM Type V] following ASTM standard test method D638 (ASTM, 1996). The specimens were die cut from thin sections (200 $\mu \mathrm{m}$ thick) that had been sliced from the UHMWPE bearings using a microtome (Collier et al., 1996). A series of the thin sections was tested to provide a depth profile of the mechanical properties. Tensile testing of the thin sections was done on a servohydraulic test machine to determine yield strength, ultimate tensile strength, modulus of elasticity and ductility (\% elongation at failure). Poisson's ratio for the layers was not determined. Cyclic tensile tests were also done to determine the fatigue strength of the thin sections of UHMWPE. The static tensile tests showed that the material in the oxidized region ('white band') had significantly lower tensile strength and very substantially reduced ductility when compared with either unsterilized material or material from layers showing less oxidation (Collier et al., 1996). A summary of the test data is given in Table 1 . It should be noted that there was some scatter in the modulus of elasticity results, so an alternative method for determining modulus of elasticity was also employed, based on oxidation measurements and on correlations by Kurtz et al. (1994, 1996) between density changes in the oxidation zone and changes in the elastic modulus. Data for modulus of elasticity from the tensile tests (Table 1) were consistent with those calculations.

The oxidized band of material has lower tensile strength than the material above or below it, but the most striking characteristic of that layer is its very substantially reduced ductility. Cyclic tensile testing showed that specimens containing a white band suffered from early fatigue failure resulting from cracks which initiated in the oxidized white band. Specimens without a white band had fatigue lives at least 10 times longer than those with the white band (Collier et al., 1996).

Based on the data reported above, it was concluded that the oxidation which resulted from gamma sterilization in air was responsible for reduced strength and ductility of the subsurface region of the UHMWPE bearings and that these deteriorated properties are related to the clinically-observed surface fatigue failures (Collier et al., 1996).

Until recently, laboratory testing of materials for TKR bearings had not demonstrated the types of surface failure that had been seen in retrieved knee bearings. Most laboratory testing had concentrated on surface wear and not on contact fatigue as a surface failure mode (Bartel et al., 1986; Walker et al., 1996), despite the prevalence of delamination and cracking in retrieved bearings. In a recent study, Currier et al. (1998) found that when neversterilized or recently-sterilized UHMWPE components were tested in a knee simulator, no cracking or delamination were observed. However, when the bearings had been gamma sterilized in air and aged prior to testing, cracking and delamination did occur under the same conditions and it was similar to the surface damage found in retrieved knee bearings (Currier et al., 1998). Thus it was concluded that in order to duplicate the clinical damage mechanisms, it is necessary to use bearing material which had been subjected to the same sterilization and aging conditions as the material of clinical knee components.

The goal of this study was to complete the picture of contact failure of UHMWPE bearings by studying the tribological conditions responsible for the initiation and propagation of damage in gamma irradiated and aged components. The study consisted of two thrusts:

- Tribotesting of UHMWPE bearing material in a knee simulator and a rolling/sliding contact tester

- Numerical analysis of the contact stress distribution in UHMWPE tibial bearings under service conditions.

Table 1 Results of tensile tests of thin sections from retrieved UHMWPE bearings (adapted from Collier et al., 1996 and Currier et al., 1998)

\begin{tabular}{lcccc}
\hline & \multicolumn{2}{c}{ Gamma sterilized in air and aged } & \\
\cline { 2 - 3 } & $\begin{array}{c}\text { Near-surface } \\
\text { 'clear' layer }\end{array}$ & $\begin{array}{c}\text { Subsurface } \\
\text { 'white' band }\end{array}$ & Center & $\begin{array}{c}\text { Unsterilized } \\
\text { (benchmark) }\end{array}$ \\
\hline $\begin{array}{c}\text { Ultimate tensile } \\
\text { strength } \\
\text { (MPa) }\end{array}$ & 35 & 20 & 27 & $55-60$ \\
$\% \begin{array}{l}\text { Elongation } \\
\text { at fracture }\end{array}$ & 230 & 15 & 70 & 550 \\
$\begin{array}{c}\text { Modulus of } \\
\text { elasticity } \\
\text { (MPa) }\end{array}$ & 600 & 1000 & 800 & 800 \\
\hline
\end{tabular}




\section{Experimental Methods and Materials}

The current study employed two different tribotest machines to assess the behavior of UHMWPE bearing material. Although some investigators have used sliding wear tests to study the wear of total knee replacement bearing materials (e.g., Walker et al., 1996), those tests do not provide the same contact kinematics as in the knee, so they usually result in surface failure modes that are different from the delamination and contact fatigue observed in clinical retrievals. In this study, two different testers, a single station knee simulator and a rolling/sliding wear tester, were used to replicate the contact fatigue failure observed in retrieved tibial bearings. This study focused on identifying these fatigue-related surface failure modes, so no quantified wear measurements (e.g., mass loss) were recorded during any of the tests. The lubricant used in all tests was distilled water. Although bovine serum has physical properties that are more similar to human synovial fluid, distilled water has been found to give a reasonable simulation of contact conditions which influence subsurface-originated rolling/ sliding contact fatigue phenomena (Currier et al., 1998).

Knee Simulator. A single station knee simulator was used to test the failure modes in the prosthesis. The knee simulator is described in detail elsewhere (Currier et al., 1998). The simulator uses actual TKR components, including a polyethylene tibial insert and a matching metallic femoral component. Load, flexion angle and cycle rate are the variables that can be controlled. All tests reported here were run with a flexion range of 0 to $35^{\circ}$. Several different load cycles were used in the test program. Although the load cycles didn't duplicate exactly the one shown in Fig. 2, the test cycles all provided a relationship between maximum load and flexion angle similar to that shown in the figure. In the most severe load cycle tested the maximum load was $2000 \mathrm{~N}$ (450 lb), whilst in the most gentle cycle the maximum load was $780 \mathrm{~N}(175 \mathrm{lb})$. Lubrication was provided by a bath of distilled water. A cooling loop placed in the bath kept the temperature in the range of $22-24^{\circ} \mathrm{C}$.

All test specimens were tibial bearings manufactured for implantation and taken from shelf inventory for testing. All were machined from UHMWPE bar stock that had been ram extruded using GUR-415 resin powder as a starting material. The components were gamma-irradiation sterilized in an ambient air environment according to the industry standard protocol of 2.5-4.0 megarads from a $\mathrm{Co}^{60}$ source. The bearings were shelf aged in air for periods ranging from 0 to $>8$ years after gamma sterilization. Tibial bearing thickness ranged from $8-18 \mathrm{~mm}$ and all were of the same design.

A single matching metallic femoral component was used for all tests in this series. It was made of cast cobalt chrome (Haynes 21 alloy), and polished according to the normal protocol by the implant manufacturer. The surface of the metallic component was cleaned with a solvent cleaner before each test, but no attempt was made to remove all adhered transfer films.

Rolling/Sliding Tester. The rolling/sliding tester enabled tests to be run at a well-controlled set of constant contact conditions, in contrast to the varying load and contact kinematics of the knee simulator. The rolling/sliding wear tester employs two counter-rotating disks to simulate the rolling and sliding in tibiofemoral loading. Details of the tester are given elsewhere (Kennedy et al., 1991). All disks were $62.5 \mathrm{~mm}$ diameter and 25 $\mathrm{mm}$ thick. The polymer test disks were machined from UHMWPE bar stock that had been ram extruded using GUR-415 polyethylene resin powder as a starting material. The UHMWPE disks were sterilized by gamma irradiation in air and artificially aged in an attempt to simulate extended time on the shelf. A number of artificial aging techniques have been developed in recent years to achieve in a short period the type of long-term oxidation damage that has been observed in shelf-aged or explanted bearings (e.g., Saum, 1994; Sun et al., 1996). Two different accelerated aging protocols were used in the study: one atmosphere of oxygen at $80^{\circ} \mathrm{C}$ for 17 days, and three atmospheres of oxygen at $70^{\circ} \mathrm{C}$ for 17 days. Control specimens were UHMWPE disks that had been neither sterilized nor aged, although some specimens which had been sterilized but not aged were also tested.

The counterface disk was machined from cobalt chrome alloy and polished to a finish similar to that of the surface of the manufactured femoral component.

The rolling/sliding tests were done at $2 \mathrm{~Hz}$ and an amplitude of $15 \mathrm{~mm}$, with a specific slide:roll ratio of 1:5. Tests were done at normal loads ranging from $135 \mathrm{~N}$ to $2000 \mathrm{~N}(450 \mathrm{lb})$. A test was continued until surface damage was first observed, or to a maximum of 2 million cycles. All tests were conducted in a distilled water bath at a temperature of $24^{\circ} \mathrm{C}$.

Post-test analysis of samples from both types of tests included optical microscopy, both before and after sectioning the component to a $200 \mu \mathrm{m}$ thickness with a microtome, and Scanning Electron Microscopy (SEM). FTIR examination of thin sections was also carried out for some specimens.

\section{Experimental Results}

Knee Simulator. Some of the knee simulator results were reported by Currier et al. (1998) and will be summarized here for completeness. Other test data have confirmed those findings.

Tests of unsterilized or recently-sterilized (but unaged) control specimens were run on the knee simulator to a maximum of 2.5 million cycles with a load cycle having a peak load of 2000 N. No cracking or delamination was found and the only wear that appeared was slight burnishing at the surface of the UHMWPE components. Measurements of the wear magnitude were not made, but the wear patterns were very similar to those found on retrieved bearings that did not suffer from cracking or delamination. Surfaces that had been aged long enough to develop an oxidized white layer, on the other hand, showed more severe surface and subsurface damage.

The sterilized and shelf aged components were run for significantly fewer cycles than the unsterilized or recently-sterilized controls (100,000 to 1 million, as opposed to 2.5 million cycles), yet the aged bearings exhibited more severe surface damage. All aged samples showed surface wear, including both flakes of polyethylene peeling from the surface and deep wear grooves on their articulating surfaces. The irradiated and aged specimens also showed surface delamination of the type found in retrieved tibial bearings. Delamination in the articular area appeared as white blister-like regions visible beneath the intact surface layer of the component (Currier et al., 1998).

After the tests the UHMWPE specimens were sliced normal to the worn surface and thin cross-sections were made by either microtoming or slicing with a diamond saw so that the subsurface region could be examined. Examination of those cross-sections revealed that most of the aged specimens had a subsurface oxidized band. The severity of the oxidation increased with the increasing aging duration after gamma irradiation. The amounts of delamination and subsurface cracking were found to increase as the severity of the subsurface oxidation increased (Table 2). Cracks in the oxidized band were more numerous beneath the contact zone on the bearings than in the non-articular region; cyclic loading and unloading appeared to affect the size and severity of the defects and cracks (Currier et al., 1998). Subsurface cracks in the components with the most severe oxidation band were quite severe and had propagated nearly to the surface (Fig. 5). The subsurface cracks seen in Fig. 5 are very similar to those observed in explanted prostheses (e.g., Blunn et al., 1991).

Rolling/Sliding Tester. The polymer specimens tested on the rolling and sliding tester produced varying degrees of wear characteristics, including burnishing, surface cracking, surface pitting, subsurface cracking and delamination. It was found that the amount of damage and the depth at which the damage developed were related to the irradiation and aging methodology (Table 3). In 
Table 2 Effect of oxidation level on results of knee simulator tests. All tests were run in distilled water at $22^{\circ}-24^{\circ} \mathrm{C}$ with maximum load of $1225 \mathrm{~N}$.

\begin{tabular}{lccc}
\hline $\begin{array}{c}\text { Subsurface } \\
\text { oxidation } \\
\text { level }\end{array}$ & $\begin{array}{c}\text { Test duration } \\
\text { (cycles) }\end{array}$ & Surface wear & $\begin{array}{c}\text { Subsurface } \\
\text { cracking }\end{array}$ \\
\hline None & 500,000 & $\begin{array}{c}\text { Wear grooves and } \\
\text { surface flaking } \\
\text { Wear grooves and } \\
\text { surface flaking }\end{array}$ & $\begin{array}{c}\text { None } \\
\text { in oxidized } \\
\text { layer }\end{array}$ \\
Severe & 500,000 & $\begin{array}{c}\text { Mild wear } \\
\text { grooves and } \\
\text { surface flaking }\end{array}$ & \begin{tabular}{c} 
Severe cracking \\
\hline
\end{tabular} \\
\hline
\end{tabular}

general, specimens that had not been sterilized showed little surface damage, even after up to 2 million cycles of oscillation. The same was true of specimens which had been sterilized but not aged after sterilization. For both types of specimens there was some light surface wear or burnishing and a small number of surface originated cracks were found on specimens which had been tested

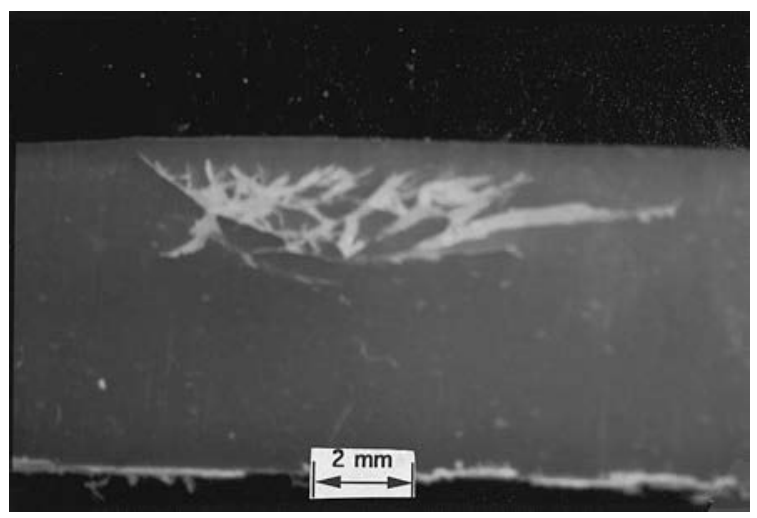

Fig. 5 Thin cross-section of tibial component tested in knee simulator for 150,000 cycles at a maximum normal load of $1225 \mathrm{~N}(275 \mathrm{lb}$.) in distilled water. It is clear that fatigue cracks have initiated at a depth of about $2 \mathrm{~mm}$ subsurface but have not yet propagated to the surface.

Table 3 Results of the rolling-sliding tests. All tests were run in distilled water at $22^{\circ}-24^{\circ} \mathrm{C}$.

\begin{tabular}{|c|c|c|c|c|}
\hline $\begin{array}{l}\text { Sterilization } \\
\text { condition }\end{array}$ & $\begin{array}{l}\text { Normal } \\
\text { load } \\
(\mathrm{N})\end{array}$ & $\begin{array}{l}\text { Test duration } \\
\text { (cycles) }\end{array}$ & Surface wear & $\begin{array}{l}\text { Subsurface } \\
\text { cracking }\end{array}$ \\
\hline Unsterilized & $900 \mathrm{~N}$ & 1 million & Burnishing & No \\
\hline Unsterilized & $2000 \mathrm{~N}$ & 2 million & $\begin{array}{l}\text { Burnishing, some } \\
\text { surface cracks }\end{array}$ & No \\
\hline $\mathrm{S}$ & $900 \mathrm{~N}$ & 1 million & Minor surface wear & No \\
\hline SA1 & $135 \mathrm{~N}$ & 140,000 & Slight burnishing & No \\
\hline SA1 & $630 \mathrm{~N}$ & 140,000 & $\begin{array}{l}\text { Surface cracks, } \\
\text { small pits }\end{array}$ & No \\
\hline SA1 & $630 \mathrm{~N}$ & 65,000 & $\begin{array}{l}\text { Some surface } \\
\text { cracks }\end{array}$ & No \\
\hline SA1 & $690 \mathrm{~N}$ & 930,000 & $\begin{array}{l}\text { Many surface } \\
\text { cracks, deep pits }\end{array}$ & No \\
\hline SA1 & $1040 \mathrm{~N}$ & 140,000 & $\begin{array}{l}\text { Surface cracks, } \\
\text { some pits }\end{array}$ & No \\
\hline SA3 & $900 \mathrm{~N}$ & 14,000 & $\begin{array}{l}\text { Subsurface cracks, } \\
\text { few spalls }\end{array}$ & Yes \\
\hline SA3 & $900 \mathrm{~N}$ & 22,000 & $\begin{array}{l}\text { Subsurface cracks, } \\
\text { spalls }\end{array}$ & Yes \\
\hline
\end{tabular}

$\mathrm{S}=$ Gamma sterilized in air, not aged.

$\mathrm{SA} 1=$ Gamma sterilized in air, aged in of one atmosphere of oxygen at $80^{\circ} \mathrm{C}$ for 17 days

SA3 = Gamma sterilized in air, aged in three atmospheres of oxygen at $70^{\circ} \mathrm{C}$ for 17 days

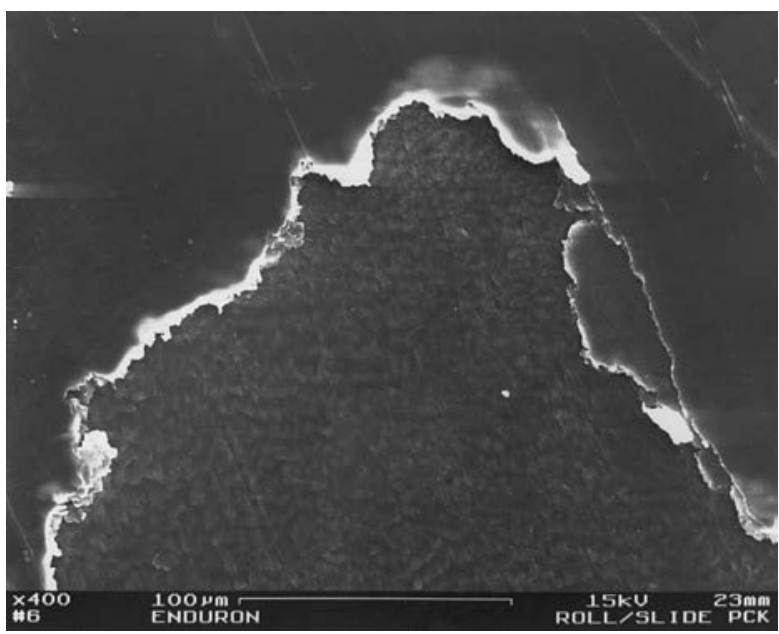

Fig. 6 SEM micrograph of surface of rolling/sliding specimen (type SA1) after 110,000 cycles at a normal load of $630 \mathrm{~N}$ in distilled water

for at least one million cycles. When cracks were found they were oriented perpendicular to the sliding direction and extended less than $1 \mathrm{~mm}$ beneath the surface. Those results were in agreement with earlier rolling/sliding tests of unsterilized UHMWPE disks (Kennedy et al., 1991).

Tests of the aged specimens (SA1 or SA3) generally resulted in cracks and/or pits on the surface, as long as the test had been carried out for a sufficient number of cycles or at high enough load (Table 3). The facts that cracks and pits did not appear in tests with low normal load and that at a given load there were more pits and cracks after a greater number of cycles are both consistent with a fatigue-type phenomenon. This is confirmation that the surface failure of the sterilized and aged UHMWPE components is related to contact fatigue.

Specimens which had been aged in one atmosphere of oxygen after sterilization (SA1) were found to have cracks and pits that originated at or very close to the contact surface. An example is shown in Fig. 6. On the other hand, specimens that were aged in three atmospheres of oxygen (SA3) showed evidence of subsurface-initiated cracks and spalls. The subsurface cracks were best viewed by slicing the specimens parallel to the sliding direction and viewing the subsurface region in cross-section. One such example is shown in Fig. 7. It is evident that a crack had initiated subsurface and had propagated approximately parallel to the surface at a depth of $300-600 \mu \mathrm{m}$.

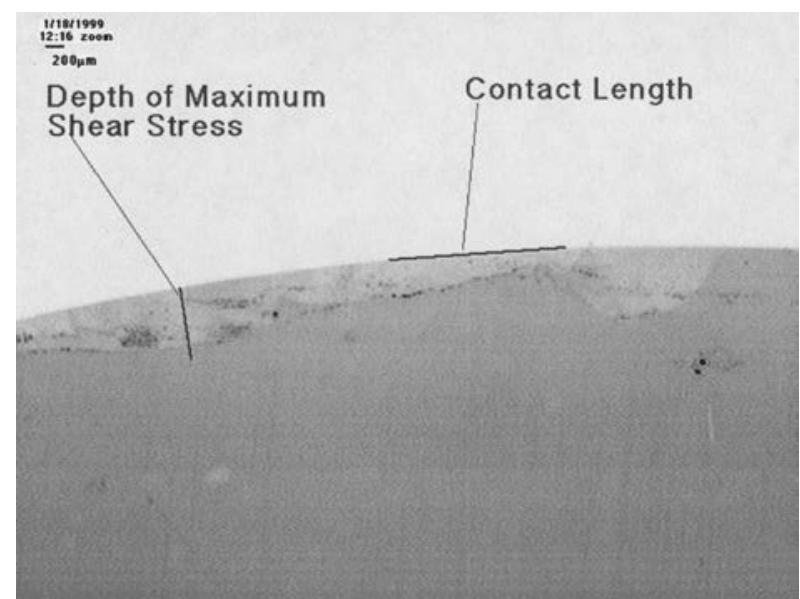

Fig. 7 Cross-section of UHMWPE disk specimen (type SA3) which had been tested for 18,000 rolling/sliding cycles at a normal load of $900 \mathrm{~N}$ in distilled water. Also shown are the analytically determined contact length (instantaneous) and depth of maximum shear stress. 


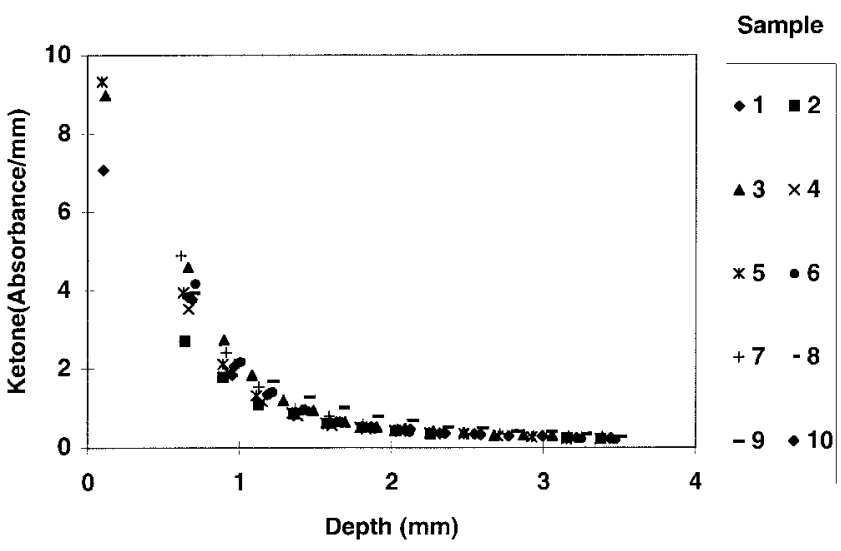

Fig. 8 FTIR-measured oxidation level (ketone peak level/thickness in millimeters) versus depth (millimeters) for UHMWPE specimens that had been aged in one atmosphere of oxygen at $80^{\circ} \mathrm{C}$ for 17 days after gamma sterilization in air (SA1)

Reasons for the differences between the SA1 and SA3 specimens became clear after they were analyzed for oxidation. Microtomed thin specimens of the disk specimens were analyzed using Fourier Transform Infrared Spectroscopy (FTIR) according to procedures that had been developed earlier for analyzing tibial bearings (Sutula et al., 1995). Oxidation profiles (ketone levels) for SA1 and SA3 specimens are shown in Figs. 8 and 9, respectively. It is apparent from Fig. 8 that oxidation in the SA1 specimens reached a peak at the surface and decreased rapidly in the subsurface region. On the other hand, the SA3 specimens had an oxidation peak beneath the surface (Fig. 9), but there was more surface oxidation than in the shelf-aged tibial bearings (Fig. 4). From these oxidation profiles, it can be concluded that in all cases the contact fatigue cracks and delamination began in the most heavily oxidized region. This region was located at a depth of about 1-2 mm for the shelf-aged bearings tested in the knee simulator, whereas it was at a depth of about $500 \mu \mathrm{m}$ for the SA3 specimens and it was at the surface for the SA1 specimens. Although there was less surface oxidation in the SA3 specimens than in the SA1 specimens, there was still considerably more surface oxidation in all accelerated-aged specimens than in shelf-aged bearings. Surfaceoriginated damage was most prevalent with the SA1 specimens, and least prevalent with the shelf-aged knee simulator specimens;

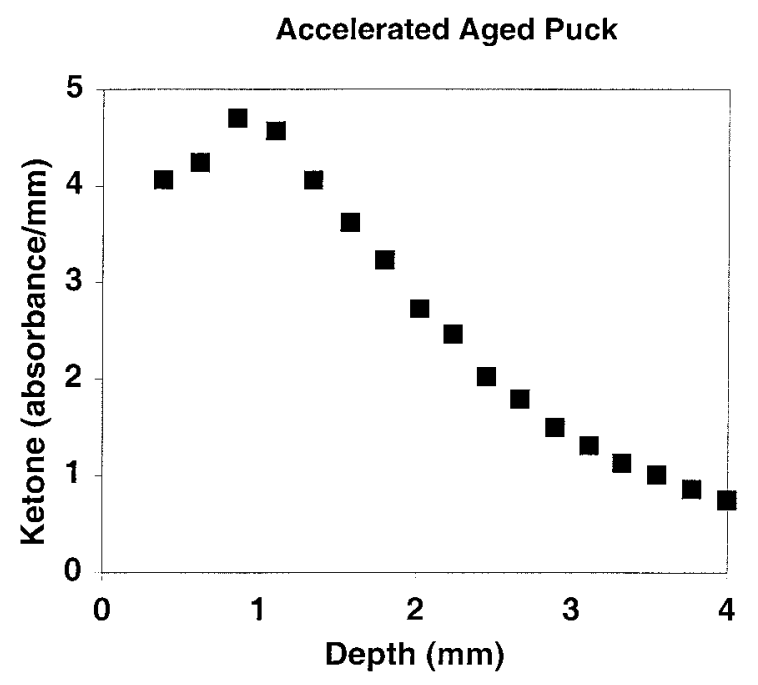

Fig. 9 FTIR-measured oxidation level (ketone peak level/thickness millimeters) versus depth (millimeters) for disk specimens that had been aged in three atmospheres of oxygen at $70^{\circ} \mathrm{C}$ for 17 days after gamma sterilization in air (SA3)

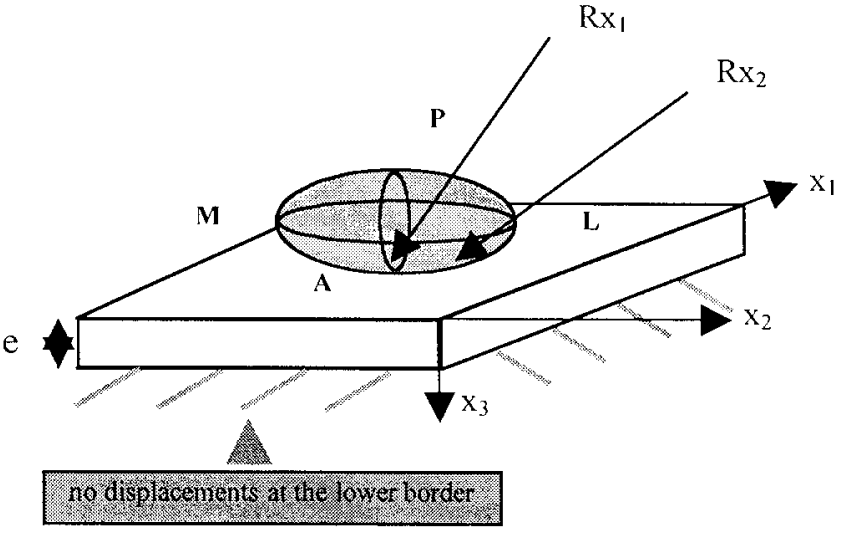

Fig. 10 Geometric model of tibial bearing for stress analysis, showing boundary conditions

therefore surface-originated damage tended to occur when there was surface oxidation.

\section{Contact Stress Analysis}

Methodology. In order to understand the conditions in the region beneath the contact where the cracks initiated, a contact stress analysis was carried out. Other investigators have studied the stress distribution in UHMWPE tibial bearings in recent years, and most have used finite element methods. For example, Sathasivam and Walker (1998) did a three-dimensional elastic finite element analysis to determine contact area, contact pressure distribution and subsurface strain energy density accumulation to predict the probability of subsurface damage in tibial bearings. Estupinan et al. (1998) and Reeves et al. (1998) did two-dimensional elastoplastic analyses of UHMWPE bearings in order to study the residual stresses and plastic strain accumulation in the contact region of the bearings. None of these analysis, however, have accounted for the fact that gamma irradiated and aged tibial bearings are inhomogenous as a result of the changes in mechanical properties that occur with oxidation. In fact, finite element methods generally require large computer times to achieve accurate stress distributions within three-dimensional non-conformal contacts with layered material properties. To overcome that problem, a combined analytical/numerical method was developed by Plumet (1998) based on integral transform techniques. That method was used in this work to study stresses in knee prosthesis bearings with layered material properties resulting from oxidation.

Details of the analytical/numerical method used here are presented elsewhere (Plumet, 1998; Plumet and Dubourg, 1998). The method is composed of the following steps:

- The UHMWPE bearing is assumed to be composed of $n$ layers. Each layer is assumed to be isotropic, linearly elastic, smooth and homogeneous, and is characterized by its elastic constants $\left(E_{i}, \nu_{i}\right)$ and thickness $e_{i}$. The properties in each layer were those determined in the tensile tests (Table 1).

- The contacting femoral condyle is assumed to be rigid and is characterized by two radii of curvature (Fig. 10). The radii of curvature are a function of the flexion angle (Fig. $1)$.

- The model is based on linear elasticity theory, unilateral contact with friction, and Fourier transforms. Fast Fourier Transforms were used to enable computational time to be reduced.

- Boundary conditions are applied as shown in Figure 10. The bottom surface was assumed to be fixed to a 'rigid' metallic backing. Contact between the condyle and the bearing was assumed to be frictionless. 
Table 4 Influence of subsurface oxidation on contact pressure and on the magnitude and depth of maximum von Mises stress

\begin{tabular}{lccc}
\hline Model & $\begin{array}{c}\text { Max. contact } \\
\text { pressure } \\
(\mathrm{MPa})\end{array}$ & $\begin{array}{c}\text { Max. von Mises } \\
\text { stress } \sigma_{v m \max } \\
(\mathrm{MPa})\end{array}$ & $\begin{array}{c}\text { Depth of } \sigma_{v m \max } \\
(\mathrm{mm})\end{array}$ \\
\hline $\begin{array}{l}\text { Unsterilized } \\
\begin{array}{c}\text { Sterilized } \\
\text { (five-layer) }\end{array}\end{array}$ & 40.3 & 12.6 & 1.6 \\
\hline
\end{tabular}

A single prosthesis geometry and knee flexion position is considered, with applied normal load $=1500 \mathrm{~N}$, total bearing thickness $=8 \mathrm{~mm}$, and radii of curvature ratio $R x_{1} / R x_{2}=0.18$

- An iterative algorithm developed by Kalkar (1990) is used to determine contact area, sliding conditions and tractions within that area, and contact pressure distribution between the rigid condyle and the tibial bearing surface.

- The surface tractions are used to determine the stress field within the multilayered body.

- The analysis was carried out at four different positions (flexion angles) and the contact radii and normal loads consistent with those positions were used.

Results. A large number of analyses were carried out to determine the influence of flexion angle, normal load, thickness of tibial bearing, interfacial friction and layer properties on the contact area, contact pressure and stress distribution. Those results are given elsewhere (Plumet, 1998); only the ones most relevant to the oxidized tibial bearing are presented here.

Analyses of homogeneous tibial bearings showed that, although the maximum normal stress was the contact pressure on the surface, the largest von Mises equivalent stress and maximum shear stress occurred within the bearing beneath the center of the contact, consistent with Hertzian contact stress analysis (Johnson, 1985). Properties for the homogeneous model were those for unsterilized UHMWPE (Table 1).

When an oxidized layer with higher modulus was included in the model, it led to smaller contact area, larger contact pressure, and an increase in von Mises equivalent stress (Table 4). For the 5-layer model, the five layers included surface 'clear' layer, subsurface oxidized layer, center region, subsurface oxidized layer, and surface 'clear' layer. Properties for each of those layers were those given in Table 1. Each layer was assumed to have the same Poisson's ratio $(\nu=0.3)$. Other conditions are given in Table 4.

The distribution of von Mises equivalent stress in a five-layer model of a tibial bearing is shown in Fig. 11 for a given normal load $(1000 \mathrm{~N})$, bearing thickness $(8.0 \mathrm{~mm})$, radii of curvature $\left(R x_{1}=60 \mathrm{~mm}, R x_{2}=130 \mathrm{~mm}\right)$, and for a semi-bent flexural

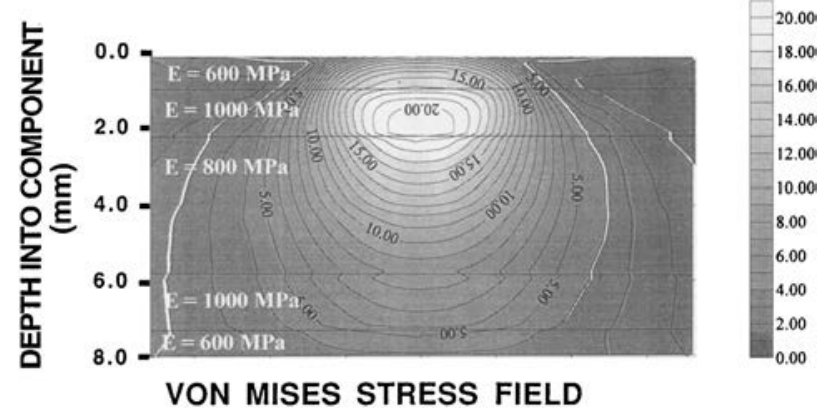

Fig. 11 Distribution of von Mises equivalent stress beneath contact with five-layer model of a particular sterilized and oxidized tibial bearing for a given normal load $(1000 \mathrm{~N})$, bearing thickness $(8.0 \mathrm{~mm})$, radii of curvature $\left(R x_{1}=60 \mathrm{~mm}, R x_{2}=130 \mathrm{~mm}\right)$, and for a semi-bent flexural position of the knee position of the knee. [It should be noted that both the prosthesis geometry (radii of curvature) and normal load are different for the case in Fig. 11 than for the one analyzed in Table 4.] It is apparent that the maximum von Mises stress occurs in the oxidized layer, which is also the layer with the lowest strength and by far the lowest ductility. This is the most probable reason for the initiation and propagation of the subsurface cracks found in retrieved tibial bearings and bearings tested in the knee simulator (Fig. 5).

It was found (Plumet, 1998) that the maximum von Mises stress increases with larger normal load, with greater knee flexion (corresponding to smaller radius of the contacting tibial component, Fig. 1), and with a decrease in the depth of the stiffer oxidized layer. It was noted above that the oxidized layer was on the surface for accelerated aging protocol SA1, was slightly subsurface (about $0.5 \mathrm{~mm}$ ) for specimens aged by protocol SA3, and was deepest (1-2 mm) for shelf aged or in-vivo aged bearings. Therefore, for given normal load and component radii, the maximum von Mises stress (or maximum shear stress) would be greater for the SA1 specimens than for SA3, and both accelerated aging cases would produce a larger von Mises stress than for bearings that had been shelf aged after gamma irradiation. Therefore, fatigue lives would be expected to be lower for the accelerated aged specimens than for shelf aged material under similar contact conditions.

The property of the oxidized material which has the most influence on the stress distribution in the layered model is its modulus of elasticity. Modulus information was not gathered for the aged specimens, so the depth of maximum shear stress shown in Fig. 7 is only approximate. However, it is clear that the von Mises stress (or maximum shear stress) will be quite high in the oxidized band, whether or not the peak value of those stresses occurs within the band (Fig. 11). It is the relatively large cyclic stress in the region of much reduced ductility and strength that is the cause of contact fatigue cracks in the oxidized band.

Another characteristic of the stress distribution in the oxidized tibial bearing which could be important is the interfacial shear stress between the layers. That interfacial shear stress could influence crack propagation at the interface, leading to delamination. It was found (Plumet, 1998) that the greatest interfacial shear stress occurs at the interface between oxidized layer and the clear layer above it, leading to the possibility of delamination at those interfaces. That result is also in accord with observations of delamination in retrieved bearings and those tested in the knee simulator.

\section{Conclusions}

Tibial bearings of total knee prostheses made from ultrahigh molecular weight polyethylene (UHMWPE) which has been gamma irradiated in air are susceptible to oxidation when aged in oxygen-containing environments. The oxidation reaches a peak about 1 to $2 \mathrm{~mm}$ beneath the surface when aged on the shelf or in-vivo. The peak oxidation takes four years or more to develop (in air or in-vivo). Accelerated aging protocols have been developed which enable oxidation to develop in a much shorter time, but the depth of the peak oxidation may differ from that in shelf-aged components, and the peak oxidation could occur on the surface under some conditions. The material properties in the oxidized layer are significantly altered, including a very substantial reduction in ductility, reductions in fatigue strength and tensile strength, and modest increases in density and modulus of elasticity.

The stress distribution beneath the contact region in a tibial bearing is affected by the presence of the oxidized layer, and the largest von Mises stress (or maximum shear stress) is generally higher than in unoxidized material. That peak stress tends to occur within the oxidized layer in naturally aged material. In accelerated aged material the peak stress may occur within or beneath the oxidized layer, depending on the location of the peak oxidation.

The high stress and reduced ductility and strength in the oxidized layer lead to the initiation of fatigue cracks in that layer. This can result in delamination, spalling, and contact fatigue failure of the UHMWPE bearings. 
Contact fatigue failure of UHMWPE bearings has been found in gamma irradiated and aged tibial bearings in a knee simulator. Those failures were very similar to those which have been noted in explanted tibial bearings. Similar contact fatigue failures have also been noted in oxidized UHMWPE material which has been tested in a rolling/sliding contact tester. The failures in material which has been aged in an accelerated manner are similar in nature to those which occurred in shelf aged bearings in the body or in the knee simulator. The difference was the shallower depth of the crack initiation in the accelerated aged material than in shelf aged bearings, and the reason for that was the fact that the oxidized layer was closer to the surface after accelerated aging than after natural (shelf) aging. No matter what the depth of the oxidized layer, the fatigue cracks appeared to always initiate in that layer.

These results strengthen the conclusions of the earlier work by Collier et al. (1996). The clinical implications are so important that all American manufacturers of total knee replacements have recently stopped using gamma irradiation in air to sterilize their UHMWPE bearings.

\section{Acknowledgment}

The authors gratefully acknowledge the support of DePuy Incorporated, a Johnson and Johnson Company, and the Veterans Administration Research and Rehabilitation Service through grant \#A-473-3DA.

\section{References}

ASTM, 1996, "Standard Test Method for Tensile Properties of Plastics," Standard D638-96

Bartel, D. L., Bicknell, V. L., and Wright, T. M., 1986, "The Effect of Conformity, Thickness and Material on Stresses in Ultra-High Molecular Weight Components for Total Joint Replacement," Journal of Bone and Joint Surgery, Vol. 68A, pp. 10411051.

Blunn, G. W., Joshi, A. B., Minns, R. J., Lidgren, L., Lilley, P., Ryd, L., Engelbrecht, E., and Walker, P. S., 1997, "Wear in Retrieved Condylar Knee Arthroplasties," Journal of Arthroplasty, Vol. 12, pp. 281-290.

Blunn, G. W., Walker, P. S., Joshi, A., and Hardinge, K., 1991, "The Dominance of Cyclic Sliding in Producing Wear in Total Knee Prostheses," Clinical Orthopaedics and Related Research, No. 273, pp. 253-263.

Cameron, H. U., 1994, Preface, Bone Implant Interface, Robert Hurley, ed., Mosby Year Book, Inc.

Collier, J. P., Sperling, D. K., Currier, J. H., Sutula, L. C., Saum, K. A., and Mayor, M. B., 1996, "Impact of Gamma Sterilization on Clinical Performance of Polyethylene in the Knee," Journal of Arthroplasty, Vol. 11(4), pp. 377-389.

Currier, J. H., Duda, J. L., Sperling, D. K., Collier, J. P., Currier, B. H., and Kennedy, F. E., 1998, "In-Vitro Simulation of Contact Fatigue Damage Found in UHMWPE Components of Knee Prostheses," IMechE Journal of Engineering in Medicine, Vol. 212H, pp. 293-302.

Estupinan, J. A., Bartel, D. L., and Wright, T. M., 1998, "Residual Stresses in Ultra-High Molecular Weight Polyethylene Loaded Cyclically by a Rigid Moving Indenter in Nonconforming Geometries," Journal of Orthopaedic Research, Vol. 16, pp. $80-88$.
ISO, 1996, "Test Methods for Laboratory Simulation of In-Vivo Wear of Total Knee Prostheses," ISO/NP 14243-1.

Johnson, K. L., 1985, Contact Mechanics, Cambridge University Press, Cambridge.

Kalkar, J. J., 1990, Three Dimensional Elastic Bodies in Rolling Contact, Kluwer Academic Publishers, Dordrecht.

Kennedy, F. E., Lee, P. H., Feehan, J. P., and Collier, J. P., 1991, "Failure of UHMWPE polymer surfaces of knee prostheses: a rolling sliding contact," Advances in Engineering Tribology, Chung Y-W and Cheng H. S., eds., STLE SP-31, pp. $100-109$.

Kurtz, S. M., Rimnac, C. M., Li, S., and Bartel, D. L., 1994, "A Bilinear Material Model for UHMWPE Components," Proc. 40th Ann. Meeting of Orthopaedic Res. Soc., p. 289.

Kurtz, S. M., Rimnac, C. M., and Bartel, D. L., 1996, "The Degradation Rate of UHMWPE in Total Joint Replacements," Proc. 42nd Ann. Meeting of Orthopaedic Res. Soc., p. 492.

Landy, M. M., and Walker, P. S., 1988, "Wear of Ultra High Molecular Weight Polyethylene Components of 90 Retrieved Knee Prostheses," Journal of Arthroplasty Supplement, Vol. 3, pp. S73-S85.

McNamara, J. L., Collier, J. P., Mayor, M. B., and Jensen, R. E., 1994, “A Comparison of Contact Pressures in Tibial and Patellar Total Knee Components Before and After Service In-Vivo," Orthopaedics and Related Research, No. 299, pp. 104-113.

Plumet, S., 1998, "Modelisation d'un milieu multicouche 3D sous sollicitations de contact: Application aux prosthèses de genou stérilisées," Doctoral thesis, INSA, Lyon France.

Plumet, S., and Dubourg, M. C., 1998, "A 3D Model for a Multilayered Body Loaded Normally and Tangentially against a Rigid Body: Application to Specific Coatings," ASME Journal of Tribology, Vol. 120, pp. 668-676.

Reeves, E. A., Barton, D. C., FitzPatrick, D. P., and Fisher, J., 1998, "A TwoDimensional Model of Cyclic Strain Accumulation in Ultra-High Molecular Weight Polyethylene Knee Replacements," IMechE Journal of Engineering in Medicine, Vol. 212H, pp. 189-198.

Sathasivam, S., and Walker, P. S., 1998, "Computer Model to Predict Subsurface Damage in Tibial Inserts of Total Knees," Journal of Orthopaedic Research, Vol. 16, pp. $564-571$.

Saum, K. A., 1994, "Oxidation Versus Depth and Time for Polyethylene Gamma Sterilized in Air," Proc. 40th Ann. Meeting of Orthopaedic Res. Soc., p. 237.

Seireg, A., and Arvikar, R. J., 1975, "The Prediction of Muscular Load Sharing and Joint Forces in the Lower Extremities During Walking," Journal of Biomechanics, Vol. 8, pp. 89-102.

Sperling, D. K., 1997, "Mechanical and FTIR Analysis of Gamma Irradiated UHMWPE," Master of Science thesis, Thayer School of Engineering, Dartmouth College, Hanover, $\mathrm{NH}$.

Sun, D. C., Stark, C., and Dumbleton, J. H., 1996, "Development of an Accelerated Aging Method for Evaluation of Long-Term Irradiation Effects on Ultrahigh Molecular Weight Polyethylent Implants," Irradiation of Polymers, R. L. Clough and S. W. Shalaby, American Chemical Soc., Vol. 620, pp. 340-349.

Sutula, L. C., Collier, J. P., Saum, K. A., Currier, B. H., Currier, J. H., Sanford, W. M., Mayor, M. B., Wooding, R. E., Sperling, D. K., Williams, I. R., Kasprzak, D. J., and Surprenant, V. A., 1995, "Impact of Gamma Sterilization on Clinical Performance of Polyethylene in the Hip," Orthopaedics and Related Research, No. 319 , pp. $28-40$

Walker, P. S., Blunn, G. W., and Lilley, P. A., 1996, "Wear Testing of Materials and Surfaces for Total Knee Replacements," Journal of Biomedical Materials Research, Vol. 33, pp. 159-175.

Williams, I. R., Mayor, M. B., and Collier, J. P., 1998, "The Impact of Sterilization Method on Wear in Knee Arthoplasty," Clinical Orthopaedics and Related Research, No. 356, pp. 170-180. 